\title{
Small-Molecule-Triggered Manipulation of DNA Three-Way Junctions
}

\author{
Isabelle T. Seemann, Vijay Singh, Mykhailo Azarkh, Malte Drescher, and Jörg S. Hartig* \\ Department of Chemistry and Konstanz Research School Chemical Biology, University of Konstanz, 78457 Konstanz, Germany
}

Supporting Information

ABSTRACT: DNA three way junctions are frequently used in nanoarchitectures. Ligand dependent designs that pro vide well characterized building blocks for structure switch ing DNA nanodevices are presented.

$\mathrm{D}$

NA has been proved to be a very versatile material for the programmed construction of nanoarchitectures. By exploita tion of Watson-Crick complementarity, it is possible to design two and three dimensional self assembling nanostructures. ${ }^{1}{ }^{3}$ A variety of confined objects ${ }^{4} 7$ and extended arrays ${ }^{8}{ }^{13}$ as well as molecular devices such as nanomachines ${ }^{14,15}$ and logic gates ${ }^{16} \quad 18$ have been realized. Environmental changes such as buffer conditions or the addition of a DNA fuel strand have been used for reversible shifting between different conformational states. ${ }^{13,19}{ }^{21}$ Moreover, aptamer-ligand interactions have been used to control the forma tion of nucleic acid based architectures. ${ }^{19,22,23}$

In the early 1980s, Seeman and co workers demonstrated pioneering work on the construction of DNA architectures with mainly three and four arms. ${ }^{24}$ Branched structures in general offer a unique window into DNA nanotechnology because more sophisticated structures can be assembled, as opposed to the sole use of one dimensional DNA objects. DNA three way junctions (DNA TWJs) have been used several times for the design of nanoscale assemblies because of their branched structure and conformational versatility. ${ }^{22,25}{ }^{27} \mathrm{In}$ addition, DNA TWJs have been characterized thoroughly with respect to their conforma tions. They show a strong tendency for pairwise coaxial stacking of two helical arms. ${ }^{28}$ It has been shown that in a perfect, fully base paired DNA TWJ, coaxial stacking of two helices is not possible without disruption of base pairing. ${ }^{29}$ DNA TWJs with fully paired nucleotides form only the open, nonstacked con formation. Introduction of a single stranded region at the branch point provides additional flexibility, and two alternately stacked conformations can be adopted in addition to the open conforma tion (see Figure 1A). ${ }^{28}$ Moreover, the sequences surrounding the junction play an important role for TWJ structure formation. In most cases, one of the stacked conformers is predominant. ${ }^{30}$ Two rules that generally reproduce the experimentally known con formational preferences have been proposed: the pyrimidine rule and the loop rule. ${ }^{31}$ In addition to the mentioned unpaired nucleotides in the branch, a stable quasi hairpin loop ( $5^{\prime}$ YTTR $3^{\prime}$, where $\mathrm{Y}=$ pyrimidine and $\mathrm{R}=$ purine $)$ and a pyrimidine ( $\mathrm{C}$ or $\mathrm{T})$ crossover in arm $a$ at the penultimate position of the branch point are required for stable coaxial stacking (see Figure 1A). Although DNA TWJs have frequently been used as construction materials for DNA nanoarchitectures, ${ }^{32}$ to date their potential to adopt two different conformations has not been utilized to create switchable DNA nanostructures. In our opinion, DNA TWJs responding to the presence of specific ligands will be of high interest for the construction of controllable DNA nanoarchitec tures. Here we report the construction and characterization of small molecule triggered DNA devices that switch between the open and stacked conformations of a DNA TWJ.

To manipulate the TWJ conformation via addition of an interaction partner, we integrated an interacting sequence into one arm of the DNA TWJ and utilized the parameters of the loop and pyrimidine rules. In a first example, we included an ATP aptamer for ligand binding. ${ }^{33}$ To implement ligand dependent conformational changes, we envisioned the following strategy (see Figure 1B): The ATP aptamer (red) would be integrated into arm $a$ of the DNA TWJ. In the open conformer, the aptamer would be forced into an undefined structure by utilizing a blocking sequence (blue) that base pairs with the aptamer stem (green). In this open state, the pyrimidine and loop rules would not be fulfilled, as a pyrimidine base is not located in arm $a$ at the penultimate position to the branch point and a stable quasi hairpin loop is not able to form.

ATP addition would be anticipated to result in aptamer induction, triggering a sequence shift at the junction site. This conformational change should yield a construct that would obey the pyrimidine rule and also form a quasi hairpin loop, allowing coaxial stacking to occur (see Figure 1B). Although most stable coaxially stacked TWJs contain two nucleotides at the junction, longer stretches of unpaired nucleotides are well tolerated and migrate similarly in native PAGE. ${ }^{34,35}$ We used both native gel electrophoresis and spectroscopic experiments to study a poten tial ligand triggered structural change. Addition of ATP resulted in a fraction that migrated faster on a $10 \%$ nondenaturating polyacrylamide gel (see Figure $2 \mathrm{~A}$ ). To show that the faster migration did not result from the increased negative charge of the complex, we repeated the experiment with AMP. The ATP aptamer shows similar affinity to AMP and hence should be induced by AMP in a comparable way. ${ }^{33}$ As shown in Figure 2B, the concentration dependence of AMP was very similar to that observed upon ATP addition. Moreover, we observed that the complex migrated at exactly the same position for both ATP and AMP. In conclusion, a shift in the conformation of TWJ 1 could be caused by either ATP or AMP. The initiation of the structural change was specific for the respective ligand, as reactions with GTP did not shift the migration in the native gel (see Figure 2C).

To further characterize the interaction of the ligand with the DNA TWJ, we performed a chemical footprinting assay with dimethyl sulfate (DMS). DMS methylates the N7 position of guanine if it is accessible, as in duplex structures. Methylation is reduced when the accessibility of the N7 position is limited by 
A
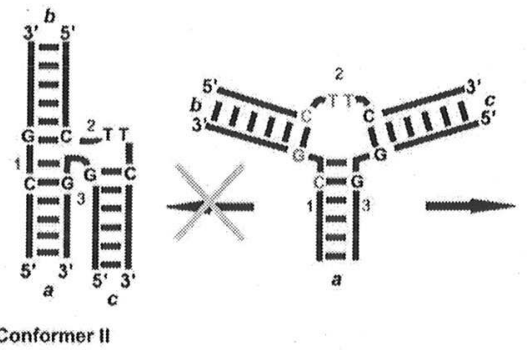

Conformer I

B

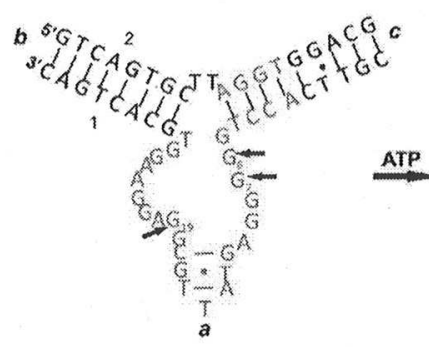

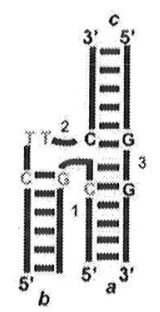

Conformer I

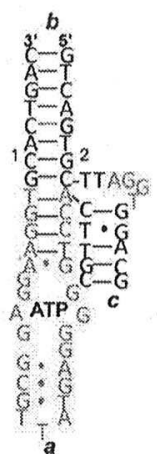

Figure 1. Isomeric forms of a DNA TWJ. The different DNA strands are numbered from 1 to 3 , with strand 2 containing unpaired bases that facilitate coaxial stacking. The double helical arms are designated as $a, b$, and $c$. (A) The pyrimidine rule and the loop rule. Important residues for conformer selection are shown: the unpaired bases (TT), the loop $\left(5^{\prime}\right.$ $\mathrm{CTT} / \mathrm{G} 3^{\prime}$ for conformer I or $5^{\prime} \mathrm{G} / \mathrm{TTC} 3^{\prime}$ for conformer II) and the penultimate position in arm $a$ ( $\mathrm{C}$ for conformer I, G for conformer II). Conformer $\mathrm{I}$ is favored, as it is in coherence with both rules. The definitions and notation follow Wu et al. ${ }^{28}$ (B) DNA three way junction device containing the ATP aptamer (TWJ 1). Green sequences are complementary. The pyrimidine and loop rules are not fulfilled in the open conformation. The ligand interaction causes the induction of the aptamer. The conformational change caused by ATP addition leads to TWJ stacking. Black arrows indicate guanine bands that disappeared upon addition of ATP in DMS footprinting experiments.

interactions with ligand, oligonucleotide, or protein. The N7 position is involved in base pairing of several guanosines (G19, G6, and G7) in the binding pocket of the ligand bound ATP aptamer. ${ }^{36}$ For TWJ 1, a nearly complete loss of those guanosine bands could be seen when ATP was added for annealing (indicated by the black arrows in Figure 1B; for the probing gel, see Figure $S 1$ in the Supporting Information). Hence, the DMS probing experiments demonstrated that the incorporated aptamer of the TWJ was not folded in the absence of ligand and that aptamer formation was induced upon addition of ATP.

Concentration dependent fluorescence resonance energy transfer (FRET) assays were performed and showed a decrease in fluorescence with increasing ATP concentration for TWJ 1 (see Figure S1B). The fluorophore FAM and the quencher BHQ 1 were attached to the $5^{\prime}$ and $3^{\prime}$ ends of the shorter DNA strand 2 . The results are in accordance with an increase in FRET quench ing upon ligand induced coaxial stacking. Comparable results have been reported elsewhere in steady state ${ }^{37}$ and time resolved ${ }^{38}$ FRET studies, in which the donor fluorescence and hence end to end distance were reduced upon coaxial stacking in DNA TWJs. To address how quickly the conformational change takes place upon ligand addition at room temperature, we carried out kinetics
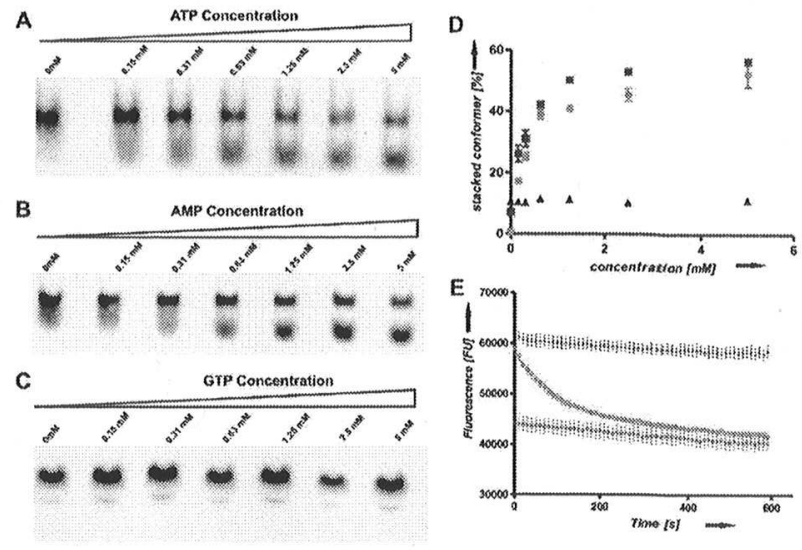

Figure 2. Ligand dependent switching of TWJ 1 containing the ATP aptamer. (A C) Nondenaturating PAGE (10\%) showing switching of TWJ 1 dependent on the concentration of (A) ATP, (B) AMP, and (C) GTP. (D) Quantification of the gels shown in (A C): red, ATP; blue, AMP; black, GTP. (E) Kinetics of fluorescence labeled TWJ 1: black, without ATP; red, addition of ATP after annealing; blue, annealed with ATP. Error bars show standard deviations of three independent experiments.

measurements. Figure 2E shows the results of the time dependent FRET experiment for TWJ 1. Hybridization of TWJ 1 was per formed in the absence of ATP, and the latter was then added to a final concentration of $5 \mathrm{mM}$ immediately before the kinetic measurements were started. A pronounced decrease of fluorescence was observed within the first $3 \mathrm{~min}$, indicating the conformational change from the open to the stacked conformer of TWJ 1. We determined a rate constante $k_{\mathrm{obs}}$ of $0.48 \mathrm{~min}^{1}$. In parallel, the fluorescence was measured for two controls, one annealed in the presence of ATP (blue in Figure 2E) and one without any addition of ATP (black in Figure 2E). No change in fluorescence intensity was observed for the controls during the same time span, except for a slight decrease resulting from photobleaching.

The FRET measurements confirmed that a conformational change of TWJ 1 takes place upon addition of the ligand, in accordance with an expected decrease of the end to end distance in the coaxially stacked state. However, to gain more insight into the ligand induced conformational changes, we used electron paramagnetic resonance (EPR) spectroscopy. The attachment of two nitroxide spin labels allows precise distance measurements utilizing double electron electron resonance (DEER) technique. We synthesized doubly spin labeled DNA TWJs via nitroxide phosphoramidite incorporation using solid phase oligonucleo tide synthesis as reported previously ${ }^{39}$ and determined distance constraints between arms $b$ and $c$ in TWJ 1 for two different label positions, as depicted in Figure 3. The first distance measurement was carried out between the spin labeled $T_{6}$ in strand 2 and $T_{9}$ in strand 1. As illustrated in Figure $3 \mathrm{~A}, \mathrm{~T}_{9}$ is located in arm $c$, close to the junction point for the open conformation. According to the sequence shift triggered by ATP addition, $\mathrm{T}_{9}$ is arranged in arm $a$, four bases away from the junction point in the stacked conformer. For the open conformer, we expected a more flexible structure, with larger distances between the different arms than in the stacked structure, caused by the unpaired thymidines and the unpaired bases and bulges in arm $a$. Indeed, the distance decreased when ATP was added, as indicated by black arrows 


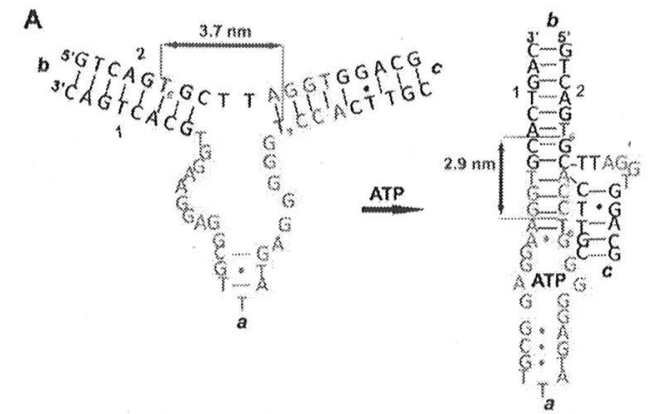

B

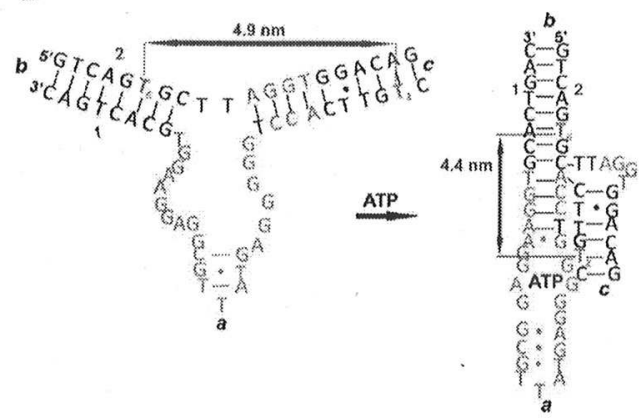

Figure 3. Distance measurements by EPR spectroscopy. (A) TWJ 1 with spin labels attached to $T_{6}$ in strand 2 and $T_{9}$ in strand 1 . (B) TWJ 1a with spin labels attached to $T_{6}$ in strand 2 and $T_{2}$ in strand 1 . The corresponding distance distribution spectra are shown in Figure S2.

in Figure 3A. In the presence of ATP, we measured a distance of $2.9 \mathrm{~nm}$ between $\mathrm{T}_{6}$ and $\mathrm{T}_{9}$. This is in very good accordance with the distance of $3.0 \mathrm{~nm}$ extracted from an existing high resolution structure of a comparable, stacked TWJ ${ }^{31}$ (see Figure S3).

In a second EPR measurement, we elongated $\operatorname{arm} c$ of TWJ 1 by introduction of an additional base pair $\left(\mathrm{A}_{19}\right.$ in strand 2 and $\mathrm{T}_{2}$ in strand 1), giving a new structure designated as TWJ 1a. The spin labels were placed at $T_{6}$ in strand 2 and $T_{2}$ in strand 1 . Again we observed a distance decrease upon ligand addition (see Figure 3B). We measured $4.9 \mathrm{~nm}$ in the absence and $4.4 \mathrm{~nm}$ in the presence of ATP. As shown in Figure S3, the distance obtained from the known TWJ structure reported by van Buuren et al. ${ }^{31}$ is $4.0 \mathrm{~nm}$. This distance distinction may originate from the completely different flanking sequences and the additional bulge formed in arm $c$ of the stacked TWJ 1. However, we still observed a distance decrease, indicating that a global conformational change involving helices $b$ and $c$ took place. In both cases, the distances measured by EPR spectroscopy decreased upon ATP addition. When the anticipated conformational change from the open conformer with wide and flexible arms to the more complex stacked conformer takes place, decreased distances are expected. Hence, the EPR distance measurements strongly indicate that stacking occurs in the ligand bound form. The EPR results are in accordance with those of the more qualitative FRET experiments.

In principle, the presented strategy of incorporating ligand responsive sequences into TWJs should be suitable for the creation of many different TWJ devices by integration of the ligand interacting sequence in different arms. Moreover, not only $a / b$ stacking but also $a / c$ stacking can be programmed. Addi tionally, the integration of different aptamers or ligand interact ing sequences provides the opportunity to create TWJ devices that can be switched by interactions with other small molecules.
To demonstrate that the concept of influencing TWJ confor mations by small molecule interactions can be generalized, we next realized a different TWJ device that contains a quadruplex forming sequence (TWJ 2). The conformational switch was programmed as follows: The quadruplex folding is inhibited in the $a / c$ stacked conformation. Upon addition of a quadruplex specific compound, induction of the four stranded structure should result in a sequence shift at the junction site and the formation of the open conformer (Figure 4A).

Again the pyrimidine and loop rules were utilized for the design. When the quadruplex is not formed, the sequence complies with the two rules, but addition of the quadruplex specific compound $360 \mathrm{~A}^{40}$ induces a fold that does not fulfill the loop rule. As Figure $4 \mathrm{~b}$ shows, a pronounced shift of the TWJ 2 construct occurred upon addition of the quadruplex specific compound 360A. Interestingly, other bisquinolinium compounds that have also been reported to be specific quad ruplex binders were able to shift the conformation as well. Addition of less selective DNA binders, derivatives of the cationic porphyrin TMPyP, did not change the migration pattern (see Figure S4).

In case of the quadruplex mediated junction, the upper band in the PAGE should represent the stacked conformer, whereas the lower one should indicate the open conformer. This observation differs from the ATP dependent TWJ 1 construct. It is very difficult to compare these two oligonucleotides because the secondary structure of the incorporated quadruplex is very unique and might have a great influence on the migration in native gels. Another decisive factor resulting in retardation could be the positive charge of $360 \mathrm{~A}$. In contrast to the ATP dependent TWJ 1 , ligand binding reduces the net charge of the complex, so slower migration in the gel is expected.

Via DMS footprinting we again confirmed that the quadruplex was formed only in the presence of $360 \mathrm{~A}$ and was not folded in its absence (see the black arrows in Figure $4 \mathrm{~A}$ and the PAGE in Figure S4). Quadruplex formation can be conveniently probed by DMS because the reactive $\mathrm{N} 7$ atoms of guanines are engaged in Hoogsteen hydrogen bonds in quadruplex structures and hence are protected from DMS methylation, whereas guanines in single stranded and duplex structures are derivatized and subsequently cleaved during the probing procedure. The DMS probing data for both ATP dependent TWJ 1 and quadruplex based TWJ 2 demonstrate that the design principles of blocking the ligand binding domain via base pairing and triggering con formational changes upon induction of the ligand bound struc tures indeed took place in the presented TWJs.

In conclusion, we have constructed and characterized DNA three way junctions that can undergo a conformational change triggered by an external stimulus: the addition of a small molecule. The TWJ based conformational switches should be well suited for incorporation into double stranded regions of higher order DNA nanostructures. This would allow for switch ing between a more rigid conformation containing coaxially stacked helices and an open conformation that would not contribute to the stabilization of the overall integrity and rigidity of the higher order structure. Experiments utilizing the presented TWJ in more complex DNA assemblies are underway. As DNA nanoarchitectures show many potential applications, such as in biosensors, food analysis, process control, environmental mon itoring, diagnostics, and therapy, ${ }^{41}$ the transfer of the presented concept to more complex nanostructures bearing further func tionalized elements will provide interesting opportunities with regard to many aspects of DNA nanotechnologies. 
A

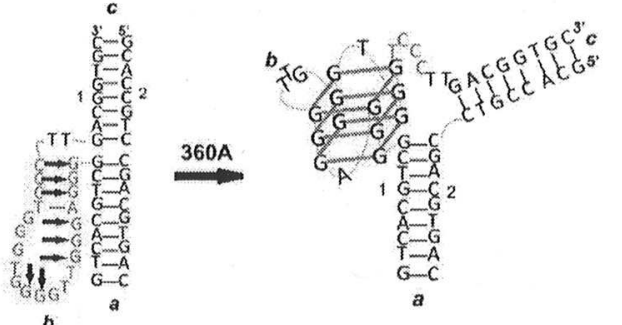

B

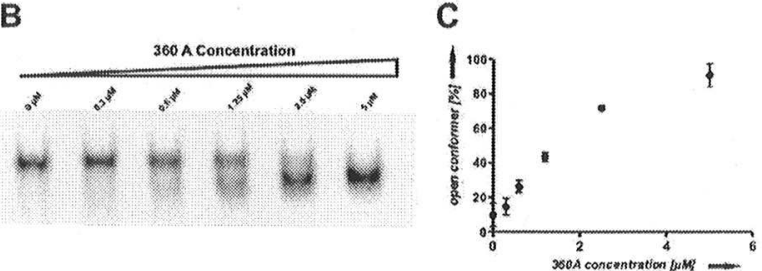

Figure 4. DNA TWJ containing a quadruplex forming sequence (TWJ 2). (A) Sequence and proposed switching mechanism of TWJ 2. The quadruplex forming sequence is highlighted in green. The $a / c$ stacked conformer is present in the absence of the quadruplex stabilizing compound. Quadruplex formation competes with a duplex that is 4 base pairs long, which can be formed by the interaction of the first $\mathrm{G}$ triplet with the blue marked bases. Addition of the quadruplex stabilizing compound $360 \mathrm{~A}$ triggers the quadruplex formation, which then results in the formation of an open TWJ. Black arrows indicate guanine residues that disappeared upon $360 \mathrm{~A}$ addition in DMS foot printing experiments. (B) Native PAGE (10\%) of TWJ 2 annealed with different $360 \mathrm{~A}$ concentrations. The intensity of the lower band accu mulates with increasing $360 \mathrm{~A}$ concentration, whereas the intensity of the upper band decreases. (C) Quantitative analysis report for the data in (B). A plot of the percentage of the formation of the open conformer against the $360 \mathrm{~A}$ concentration is shown; error bars show standard deviations of three independent experiments.

\section{ASSOCIATED CONTENT}

Supporting Information. Experimental methods and additional data. This material is available free of charge via the Internet at http://pubs.acs.org.

\section{AUTHOR INFORMATION}

Corresponding Author

joerg.hartig@uni konstanz.de

\section{ACKNOWLEDGMENT}

J.S.H. acknowledges the VolkswagenStiftung for funding of a Lichtenberg Professorship. We are grateful to Corinne Guetta and Marie Paule Teulade Fichou for providing the bisquinoli nium derivatives PhenDC and 360A. This work was financially supported by the DFG (DR 743/2 1) and the Zukunftskolleg of the University of Konstanz.

\section{REFERENCES}

(1) Seeman, N. C. Mol. Biotechnol. 2007, 37, 246.

(2) Feldkamp, U.; Niemeyer, C. M. Angew. Chem., Int. Ed. 2006, $45,1856$.

(3) Simmel, F. C. Angew. Chem., Int. Ed. 2008, 47, 5884.

(4) Chen, J. H.; Seeman, N. C. Nature 1991, 350, 631.

(5) Shih, W. M.; Quispe, J. D.; Joyce, G. F. Nature 2004, 427, 618.

(6) Rothemund, P. W.; Ekani Nkodo, A.; Papadakis, N.; Kumar, A.; Fygenson, D. K.; Winfree, E. J. Am. Chem. Soc. 2004, 126, 16344.
(7) Goodman, R. P.; Berry, R. M.; Turberfield, A. J. Chem. Commun 2004, 1372.

(8) Goodman, R. P.; Schaap, I. A.; Tardin, C. F.; Erben, C. M.; Berry R. M.; Schmidt, C. F.; Turberfield, A. J. Science 2005, 310, 1661.

(9) Park, S. H.; Barish, R.; Li, H.; Reif, J. H.; Finkelstein, G.; Yan, H.j Labean, T. H. Nano Lett. 2005, 5, 693.

(10) Park, S. Y.; Lytton Jean, A. K.; Lee, B.; Weigand, S.; Schatz G. C.; Mirkin, C. A. Nature 2008, 451, 553.

(11) Yan, H.; Park, S. H.; Finkelstein, G.; Reif, J. H.; LaBean, T. H. Science 2003, 301, 1882

(12) He, Y.; Chen, Y.; Liu, H.; Ribbe, A. E.; Mao, C. J. Am. Chem. Soc. 2005, 127, 12202

(13) Feng, L.; Park, S. H.; Reif, J. H.; Yan, H. Angew. Chem., Int. Ed. 2003, 42, 4342 .

(14) Dittmer, W. U.; Reuter, A.; Simmel, F. C. Angew. Chem., Int. Ed. 2004, 43, 3550.

(15) Mao, C.; Sun, W.; Shen, Z.; Seeman, N. C. Nature 1999, 397,144 .

(16) Mao, C.; LaBean, T. H.; Reif, J. H.; Seeman, N. C. Nature 2000, 407, 493.

(17) Seelig, G.; Soloveichik, D.; Zhang, D. Y.; Winfree, E. Science 2006, 314, 1585.

(18) Macdonald, J.; Li, Y.; Sutovic, M.; Lederman, H.; Pendri, K.; Lu, W.; Andrews, B. L.; Stefanovic, D.; Stojanovic, M. N. Nano Lett. 2006, 6,2598 .

(19) Wieland, M.; Benz, A.; Haar, J.; Halder, K.; Hartig, J. S. Chem. Commun. 2010, 46, 1866

(20) Dirks, R. M.; Pierce, N. A. Proc. Natl. Acad. Sci. U.S.A. 2004, 101,15275 .

(21) Yin, P.; Choi, H. M.; Calvert, C. R.; Pierce, N. A. Nature 2008, $451,318$.

(22) Wang, Z. G.; Wilner, O. I.j Willner, I. Nano Lett. 2009, 9, 4098.

(23) Mitchell, J. C.; Harris, J. R.; Malo, J.; Bath, J.; Turberfield, A. J.J. Am. Chem. Soc. 2004, 126, 16342.

(24) Seeman, N. C. J. Theor. Biol. 1982, 99, 237.

(25) Seeman, N. C. Curr. Opin. Struct. Biol. 1996, 6, 519.

(26) LaBean, T. H.; Yan, H.; Kopatsch, J.; Liu, F.; Winfree, E.; Reif, J. H.; Seeman, N. C. J. Am. Chem. Soc. 2000, 122, 1848.

(27) Boer, D. R.; Kerckhoffs, J. M.; Parajo, Y.; Pascu, M.; Uson, I.; Lincoln, P.; Hannon, M. J.; Coll, M. Angew. Chem., Int. Ed. 2010, 49, 2336.

(28) Lilley, D. M. Q. Rev. Biophys. 2000, 33, 109.

(29) Duckett, D. R.; Lilley, D. M. EMBO J. 1990, 9, 1659.

(30) Wu, B.; Girard, F.; van Buuren, B.; Schleucher, J.; Tessari, M.; Wijmenga, S. Nucleic Acids Res. 2004, 32, 3228.

(31) van Buuren, B. N.; Overmars, F. J.; Ippel, J. H.; Altona, C.; Wijmenga, S. S. J. Mol. Biol. 2000, 304, 371.

(32) Hamada, S.; Murata, S. Angew. Chem., Int. Ed. 2009, 48, 6820.

(33) Huizenga, D. E.; Szostak, J. W. Biochemistry 1995, 34, 656.

(34) Welch, J. B.; Duckett, D. R.; Lilley, D. M. Nucleic Acids Res. $1993,21,4548$

(35) Leontis, N. B.; Kwok, W.; Newman, J. S. Nucleic Acids Res. 1991, 19,759

(36) Lin, C. H.; Patel, D. J. Chem. Biol. 1997, 4, 817.

(37) Stuhmeier, F.; Welch, J. B.; Murchie, A. I.; Lilley, D. M.; Clegg,

R. M. Biochemistry 1997, 36, 13530.

(38) Yang, M.; Millar, D. P. Biochemistry 1996, 35, 7959.

(39) Singh, V.; Azarkh, M.; Exner, T. E.; Hartig, J. S.; Drescher, M. Angew. Chem., Int. Ed. 2009, 48, 9728.

(40) Monchaud, D.; Yang, P.; Lacroix, L.; Teulade Fichou, M. P.; Mergny, J. L. Angew. Chem., Int. Ed. 2008, 47, 4858.

(41) Abu Salah, K. M.; Ansari, A. A.; Alrokayan, S. A. J. Biomed. Biotechnol. 2010No. 715295 . 